\title{
Seventeen years of prenatal magnetic resonance imaging at the Institute of Mother and Child in Warsaw
}

\author{
Monika Bekiesińska-Figatowska ${ }^{A, B, C, D, E, F, G}$, Anna Romaniuk-Doroszewska ${ }^{B, C D}$, Hanna Brągoszewska ${ }^{B, D}$, \\ Beata Iwanowska ${ }^{B, D}$, Sylwia Szkudlińska-Pawlak ${ }^{B, D}$, Jarosław Mądzik ${ }^{B, D}$, Marek Duczkowski ${ }^{B, D}$, \\ Katarzyna Krupa ${ }^{B, D}$, Astra Cabaj ${ }^{B, D}$, Piotr Kwaśniewicz ${ }^{B, D}$, Agnieszka Duczkowska ${ }^{B, D}$ \\ Department of Diagnostic Imaging, Institute of Mother and Child, Warsaw, Poland
}

\section{Abstract}

Purpose: The purpose of this paper is to summarise and to present the experience of the main Polish centre for prenatal magnetic resonance imaging (MRI) and to discuss the place and role of MRI in antenatal diagnosis, management, and counselling.

Material and methods: Retrospective analysis of the examinations performed in the years 2001-2017.

Results: In total, 1221 medical records and/or image files were collected. The full documentation of ultrasonography and MRI referrals, reports, and images was not available in every case. During the first three years 98 pregnant women were examined (approximately 33 per year, one study per fortnight). After purchase of own MR scanner, the number of examinations grew constantly, reaching 208 in 2017, which means almost one per day, and the highest number was eight in one day. We examined 45 pairs of twins, including three pairs of conjoined twins.

Conclusions: MRI at our Institute is a practically used second-line foetal imaging tool, necessary to confirm, complete, or correct sonographic diagnoses, with important impact on clinical decisions concerning management of pregnancy and of the neonate, termination of pregnancy, choice of the time, place, and mode of delivery, and neonatal care, as well as on counselling. In experienced hands, MRI is to date the last and the best non-invasive method of diagnosis in utero. It is frequently requested by the interdisciplinary team for foetal diagnosis and therapy and routinely performed in everyday practice.

Key words: foetus, magnetic resonance imaging (MRI), birth defects.

\section{Introduction}

The experience of the Department of Diagnostic Imaging of the Institute of Mother and Child in the field of foetal magnetic resonance imaging (MRI) dates back to the beginning of the twenty-first century, although the first examinations were performed outside the Institute under a grant of the Committee for Scientific Research and evaluated at the Institute. We failed to collect and count all the performed studies, but the number of those collected and counted reaches over 1000 up to the end of the year 2017. During this time the frequency of referrals for foetal MRI increased many-fold: from one every few weeks to as many as eight per day. Given the above, the authors do not hesitate to call themselves the most experienced centre for prenatal MRI in Poland. This experience has resulted in numerous publications in scientific journals [1-13] and books [14,15], and the release of our own Atlas of Foetal Anomalies in co-operation with Jagiellonian University in Krakow [16]. However, up till now, there has been no comprehensive

\footnotetext{
Correspondence address:

Monika Bekiesińska-Figatowska, Department of Diagnostic Imaging, Institute of Mother and Child, 17a Kasprzaka St., 01-211 Warsaw,

phone: +4822327 71 56, fax: +4822327 71 95, e-mail: zaklad.rtg@imid.med.pl
}

Authors' contribution:

A Study design - B Data collection · C Statistical analysis · D Data interpretation - E Manuscript preparation · $F$ Literature search · G Funds collection 
publication in the Polish literature about Polish experience in prenatal MRI. Our experience also results in the fact that new centres in Poland trying to start work in the field of prenatal MRI consult our opinion on the protocol and how to conduct the studies and consult the obtained images. We meet the criteria set by the International Society of Ultrasound in Obstetrics and Gynaecology (ISUOG) in the recent publication [17] to qualify as a teaching centre, i.e.: 1) we have multidisciplinary specialists working in the field, including, and not limited to, foetomaternal specialists, radiologists, and obstetricians; 2) we have institutional experience, with at least 500 foetal MRIs and at least two examinations performed per week; 3 ) we have published papers and reference material in this field.

The purpose of this paper is to summarise and to present the experience of the main Polish centre for prenatal magnetic resonance imaging and to discuss the place and role of MRI in antenatal diagnosis, management, and counselling.

\section{Material and methods}

The available documentation of foetal MR examinations performed at the Department of Diagnostic Imaging of the Institute of Mother and Child in Warsaw since 2004 and before that year at other centres involved in research project No. 6 PO5E 071 21, was retrospectively reviewed and analysed. Institutional Bioethics Committee approval was not required due to the retrospective nature of this study. In each case written, informed consent for clinical MRI was obtained from the patients and/or their legal guardians.

\section{Results}

In total, 1221 medical records and/or image files were collected. However, the full documentation (ultrasonography report and images and referral sheet for MRI and MR images and report) was not available in every case.

During three years of research project No. 6 PO5E 071 21 , titled "The value of prenatal magnetic resonance imaging in the diagnosis and treatment of foetal and neonatal abnormalities", 98 pregnant women were examined (approximately 33 per year, one study per two weeks). After purchase of our own MR scanner, the number of performed examinations grew constantly every year, reaching 208 in 2017, which means that nowadays we perform prenatal studies almost every day and the highest number of examined women in one day was eight.

We examined 45 pairs of twins, including three pairs of conjoined twins.

\section{Discussion}

Although ultrasonography (US) is and will remain the first-line imaging method in the assessment of the foetus, its well-known limitations have made MRI - a long time ago - a very useful and well-recognised adjunct to US. These limitations of US include foetal and placental position, advanced foetal age, oligohydramnios, and maternal obesity, which do not pose problems to MRI [18], although MRI in turn is not useful in early pregnancy with a small and constantly moving foetus [19]. The advantages of MRI include high tissue resolution and large field of view [20]. MRI has gone far beyond T2-weighted images only, and many other imaging sequences are used providing a large amount of complementary information about normal and abnormal foetal tissues [21]. Single-shot fast spin-echo T2-weighted images (SSFSE/T2) acquired each as a single slice are the mainstay of foetal MRI, being the least dependent on foetal motion and depicting anatomy [22]. The other sequences are based on simultaneous acquisition of all the slices, which makes them much more dependent on movement, however, sedation is not necessary and is not used for foetal MRI [23]. These other sequences include fast spin echo T1-weighted images (FSE/T1), fat-saturated T1-weighted images (LAVA), two-dimensional fast imaging employing steady-state acquisition (FIESTA/2D), echoplanar gradient echo (GRE EPI), diffusion-weighted imaging (DWI), and susceptibility-weighted imaging (SWI) in our everyday practice. FSE/T1 and LAVA are useful in the evaluation of liver, thyroid gland, and meconium-filled bowel as well as depicting haemorrhage, calcifications, and fat $[22,24,25]$. FIESTA/2D gives a very comprehensive overview of foetal anatomy and provides good quality images of the heart. GRE EPI shows bony and cartilaginous elements of the skeleton. SWI is sensitive to bleeding. DWI provides information on brain ischaemia and also on the function of foetal kidneys [26].

The method is safe and not contraindicated even in the first trimester of pregnancy, although it is not useful and not used in this period for foetal indications for the reasons mentioned above [23]. We usually perform MRI not earlier than at the gestational age of 18-19 weeks, to be able to visualise details of anatomy and pathology.

The contraindications to MRI include pacemakers and ferromagnetic foreign bodies, as well as some medical implants within the mother's body.

The patient does not require any specific preparation for the examination except certain elements that can help to reduce foetal motion, e.g. at least four hours of fasting and avoiding caffeine-containing drinks (coffee, Coca-Cola) before the examination. The patients lie on their back or - in late pregnancy - on their back but with the right side elevated to avoid compression of the inferior vena cava, and they can lie in the entirely left lateral position if it is more comfortable [23] although, depending on the size of the mother's belly, in this position the surface coil's coverage may be insufficient for the whole foetus.

Gadolinium-based contrast media are not used in foetal MRI although they may be used for maternal indications if the potential advantages overweigh the potential risk. 
SSFSE/T2, GRE EPI, DWI, and SWI are performed during free breathing; some sequences (FIESTA/2D, FSE/T1, LAVA) require breath hold not lasting longer than 13 seconds per acquisition, which is in most cases well tolerated by the mothers.

One of the most important points is the experience of the centre performing foetal MRI. There are no routine study protocols, each subsequent sequence is planned on the basis of the previous one. The choice of the sequence and of the imaging plane depends on the suspected pathology. The radiologist must be familiar with foetal anatomy, which changes with age and with numerous congenital anomalies and syndromes. The radiologist must be present in the MR unit during the whole study and must continue to plan the next sequences.

As for the experience, one should bear in mind that it ranged from zero at the beginning of our work in this field to the present state. We do not report the overall percentage of MRI findings that have changed the final diagnosis to a greater or smaller extent, the percentage of inconclusive studies, and the percentage of studies that did not provide significant new information for the diagnosis as compared to US because the full documentation (US images and report, referral sheet for MRI, MR images and report) is not available in every case. We started with a lack of experience and limited range of available sequences. With time and growing armamentarium, the number of referrals grew as well, reflecting: 1) the growing conviction of gynaecologists about the value and need for foetal MRI; 2) the constantly corrected and improved study protocol, allowing the detection of more and more fine details; and 3) the increasing experience of the team of radiologists and technologists.

The indications for foetal MRI are "to confirm, correct, or complete prenatal ultrasound diagnosis" [27], so the organ/system suspected of pathology must primarily be studied, but assessment of the entire body of the foetus is obligatory.

The evaluation of the central nervous system (CNS) was historically the first indication to perform foetal MRI. During development in utero the brain changes from the smooth tissue mass with wide fluid spaces to the appearance that we see after birth: with adequate gyration and sulcation and narrower ventricles. Ventriculomegaly is one of the most frequent indications for foetal MRI in the search for accompanying malformations, and in most cases it remains an isolated finding (which explains the relatively high percentage of MR examinations that do not add new knowledge about the foetus in these cases), but in part of them MRI shows additional anomalies that were occult to US. Knowledge of the order of appearance of gyri and sulci is essential for the assessment of brain maturity, as well as knowledge of layering of brain parenchyma with the progress of neuronal migration [6]. The latter is reflected by changes of signal intensity (SI) on T1-, T2-, and diffusion-weighted images (T1WI, T2WI, DWI). Myelination also causes changes of SI on T1WI and T2WI and is best seen close to the end of pregnancy in the posterior limbs of the internal capsules. The corpus callosum is visualised directly in the midsagittal slice on brain MRI. Also, the structures of the posterior cranial fossa can be seen directly, assessed in terms of size, shape, and position, and measured. MRI depicts tiny structures like optic nerves and hypophysis. That is why sonographically occult abnormalities can be detected on foetal MRI. These anomalies include, among others, malformations of cortical development, callosal agenesis/hypoplasia, pontine and cerebellar hypoplasia/dysplasia, tuberous sclerosis, and also the acquired lesions, such as periventricular leukomalacia, porencephaly, and cerebral and cerebellar haemorrhages [24]. The latter require differentiation of mass lesions, which is also possible on foetal MRI thanks to analysis of the combination of SI from various imaging sequences, allowing for diagnosis of calcifications, fat, blood products, and solid and cystic elements. The above-described possibilities offered by MRI allowed us, among others, to correctly identify Walker-Warburg syndrome as early as at the gestational age (GA) of 22 weeks on the basis of kinking of the brain stem (Figure 1), as well as benign and malignant congenital brain tumours, e.g. craniopharyngioma [4,7]. The complications of monochorionic pregnancy, such as abnormal cortical folding in the living recipient in twin-twin transfusion syndrome (TTTS) or conjoined twins with the assessment of possible separation are also good examples.

Although the assessment of the vertebrae, detection of their defects, and determination of the level of anomalies

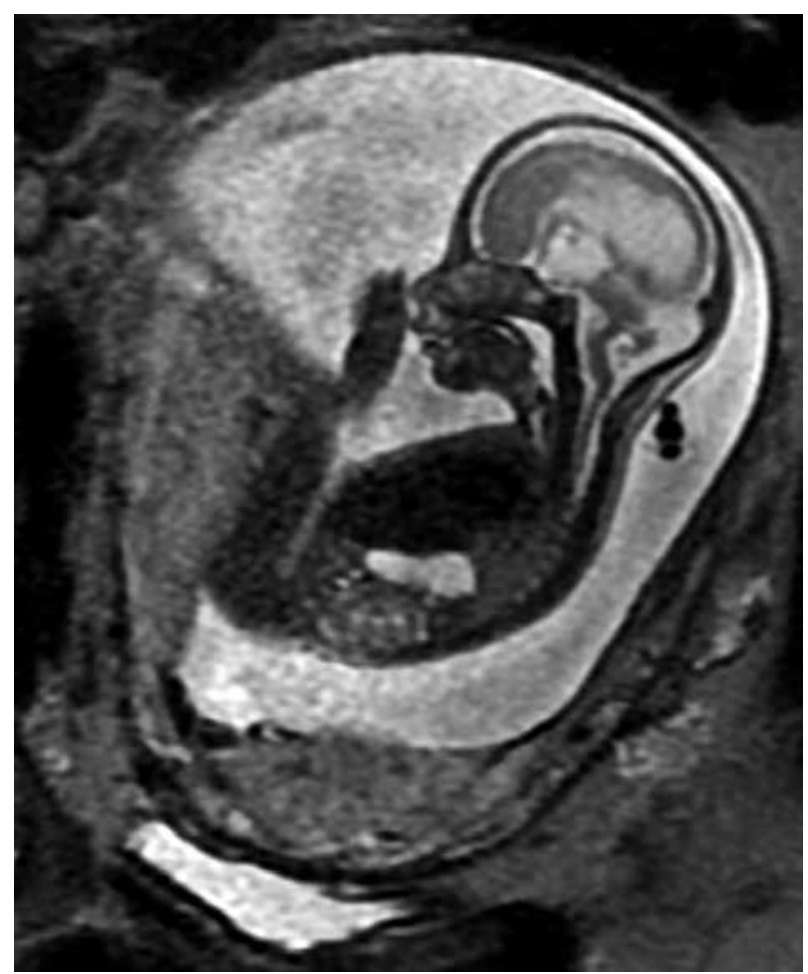

Figure 1. $22 \mathrm{Hbd}$. SSFSE sequence, T2-weighted images. Abnormal brain stem with kinking and cerebellar hypoplasia in Walker-Warburg syndrome 

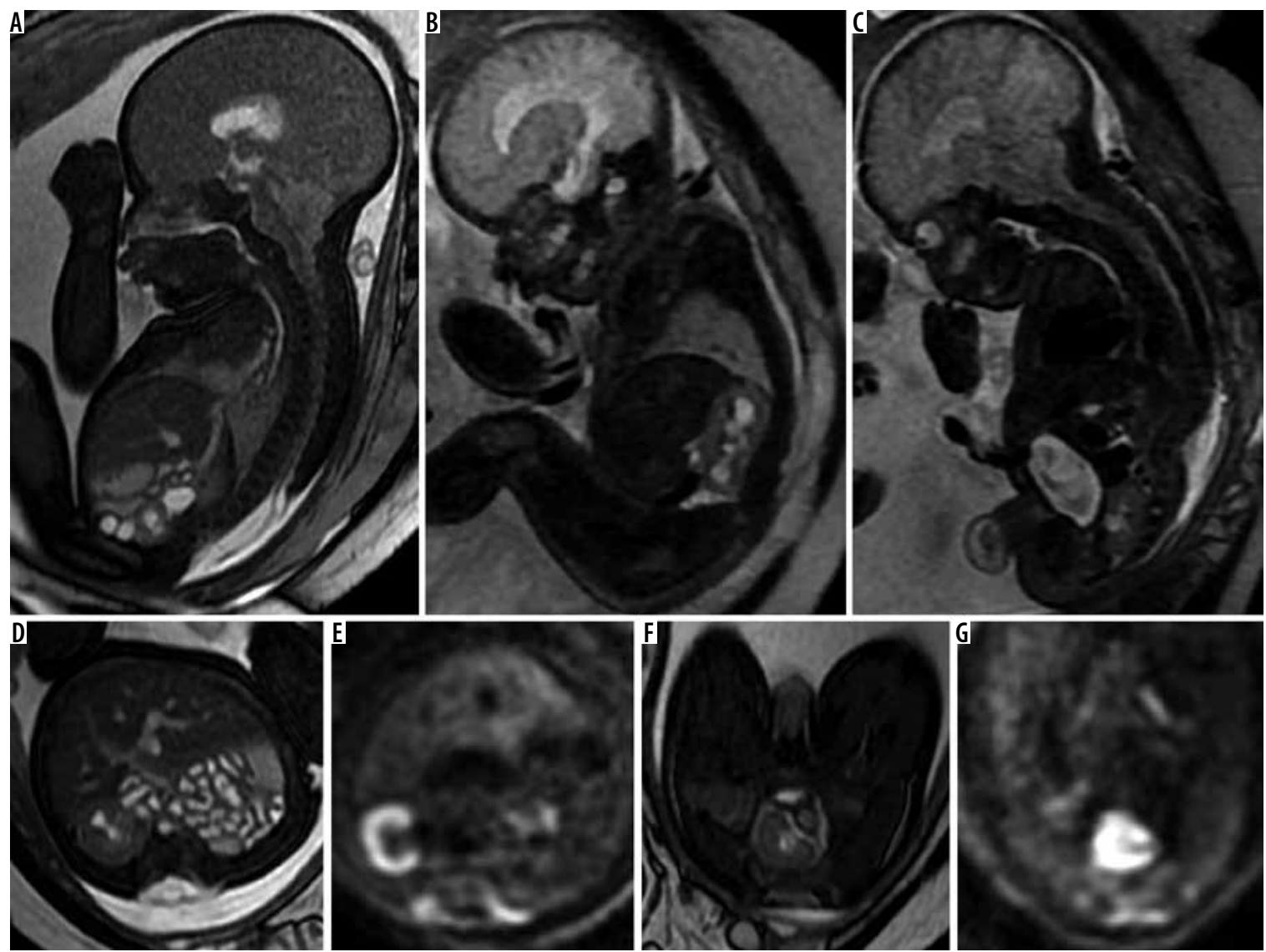

Figure 2. $35 \mathrm{Hbd}$. A, D, F) FIESTA/2D; B, C) SSFSE sequence, T2-weighted images. Arnold-Chiari malformation: myelomeningocele in the region from thoracic-lumbar junction to sacral part of the vertebral column, small posterior cranial fossa with invisible fluid spaces and hindbrain herniation (A). The right kidney is normally placed (B, D, E), while the left one - invisible on ultrasound - is in pelvic, retrocystic location (C, F, G). High signal intensity of both kidneys on diffusion-weighted imaging $(\mathrm{E}, \mathrm{G})$

in the vertebral column remains the domain of ultrasound, the introduction of the GRE EPI sequence made it much easier to separate vertebral bodies and intervertebral spac-

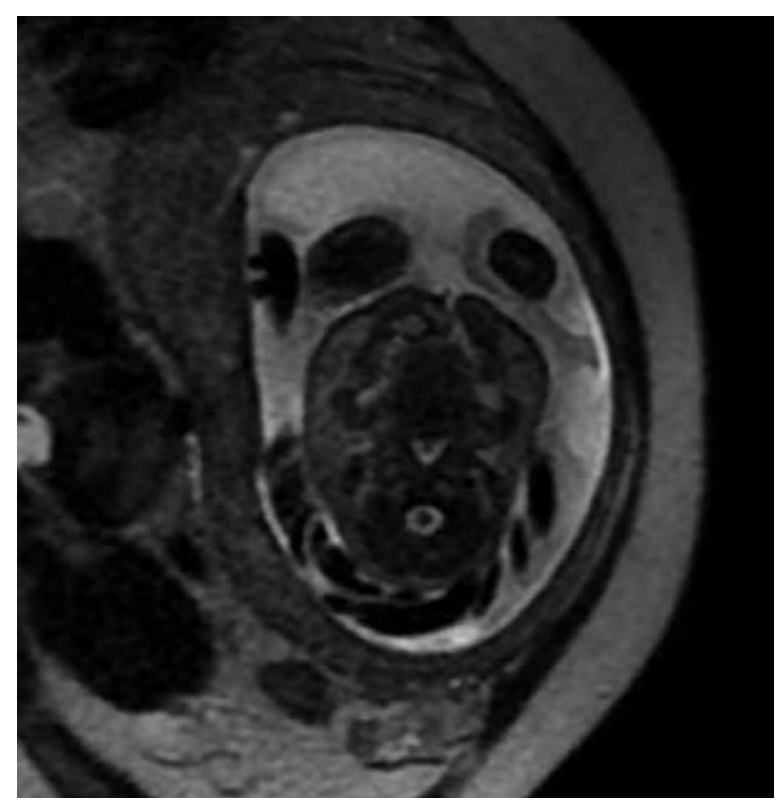

Figure 3. $32 \mathrm{Hbd}$. SSFSE sequence, T2-weighted images. Unilateral cleft lip and alveolus on the left side es on MRI. Evaluation of the contents of the spinal canal is the domain of MRI [5]. All types of Chiari malformation can be diagnosed correctly on MRI (Figure 2A); moreover, MRI is mandatory before deciding on surgery in utero, which is also performed in Poland. It is used for postoperative follow-up as well [10].

In the region of the face and neck MRI is used in the evaluation of the most common congenital craniofacial anomaly, which is cleft lip and/or palate (Figure 3). It shows the connections between the oral and nasal cavities, the degree of involvement of the secondary palate, and the lateral and anterior-posterior extent of the cleft better than US [12]. The above-mentioned analysis of the combination of SI in various MR sequences allows - just like in the brain and other parts of the body, which will be mentioned again below - differentiation of pathological masses in this area. Characteristic T1-hyperintense goitre is easy to differentiate from other solid (e.g. teratomas) and cystic (hygromas) masses. MRI enables us to rule out or to confirm meningoencephalocele and to differentiate it from cystic hygroma [28]. The fluid-filled airways are T2-hyperintense, and therefore their obstruction by the face and neck masses is relatively easy to demonstrate on MRI, thus helping to decide on ex utero intrapartum treatment (EXIT). US is frequently incapable of depicting 

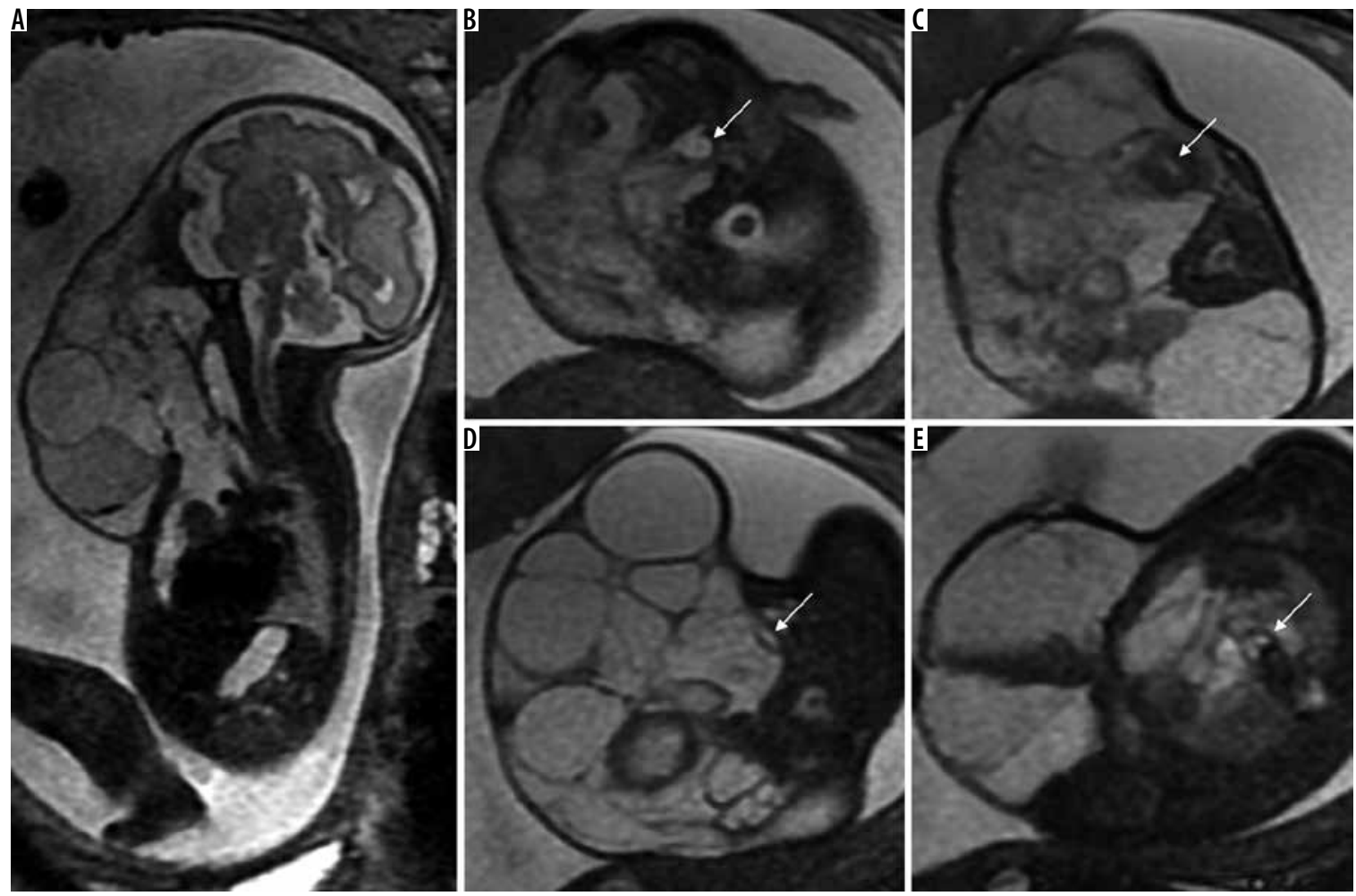

Figure 4. $29 \mathrm{Hbd}$. SSFSE sequence, T2-weighted images. Giant venolymphatic malformation extending over head, neck, and chest of the foetus (A), displacing and compressing the throat (B), larynx (C), trachea (D), and its bifurcation (E)

details of the course of the airways, as in our case of giant venolymphatic malformation, with their extreme compression and displacement (Figure 4). MR images were indispensable for the anaesthesiologist to prepare for the difficult intubation after birth. In another case, of the facial hemangiopericytoma, an unusual tumour in this age group and in this location, US showed a pedunculated tumour deriving from the nasal cavity, while on MRI it was broad-based on the face, and both nasal cavities were free and patent, which was confirmed during surgery.

As in case of brain maturation, foetal MRI allows us to follow the natural development of the lungs, which display increasing T2-hyperintensity and volume with age. Therefore, small and only faintly T2-hyperintense lungs reflect pulmonary hypoplasia [29]. Lung volumetry on MRI is used to predict the outcome in foetuses with pulmonary hypoplasia and before deciding on therapeutic options such as extracorporeal membrane oxygenation (ECMO) [30]. The black line of the diaphragm clearly separates thoracic and abdominal organs and helps to differentiate pulmonary pathology from congenital diaphragmatic hernia $(\mathrm{CDH})$. Strongly T1-hyperintense - bright (and T2-hypointense - dark) meconium filling the bowel loops located in the thorax is a perfect marker of $\mathrm{CDH}$ (Figures 5A-B) leaving no doubt as to the fact that this is not congenital pulmonary airway malformation (CPAM), which shows an inverse SI: T1-hypo- and T2-hyperintensity. A lack of possibility of sonographic differentiation of bowel only containing $\mathrm{CDH}$ and CPAM is often the reason to refer the patient to MRI, and this method provides a straightforward diagnosis. Correct diagnosis is crucial for the implementation of treatment by means of foetoscopic endotracheal balloon occlusion (FETO) - this method has already been introduced in our country [31]. It is also easy to evaluate the position of the liver, which is characteristically T1-hyperintense, correctly under or incorrectly over the diaphragm (Figure 5B). SI analysis usually leads to correct diagnosis of thoracic teratomas as well.

The heart, as a "black hole" on SSFSE/T2WI, has not been initially evaluated by MRI beyond the aspect of size and position and the presence of tumours, mainly rhabdomyomas. With the advent of "white blood" sequences, such as FIESTA/2D (Figure 6), assessment of cardiac walls and chambers is feasible. As stated in the recent paper from the American Heart Association, "MRI has the potential to complement ultrasound imaging in the evaluation of foetal visceroatrial situs, cardiac structure, and cardiac function. Although the clinical utility of the technology has not been well established, and although it is currently used mostly as a research tool, foetal cardiac MRI is reasonable to perform in the evaluation of certain forms of foetal cardiovascular disease, including heterotaxy and systemic venous anomalies" [32]. In our centre we do not perform foetal cardiac MRI as such.

Some characteristic MRI features of abdominal organs have already been mentioned above. As far as the appearance of bowel loops is concerned, one has to be familiar with the natural history of their development and meconium production because it influences SI on MR images [16,33]. Fluid-filled structures, such as stomach, 

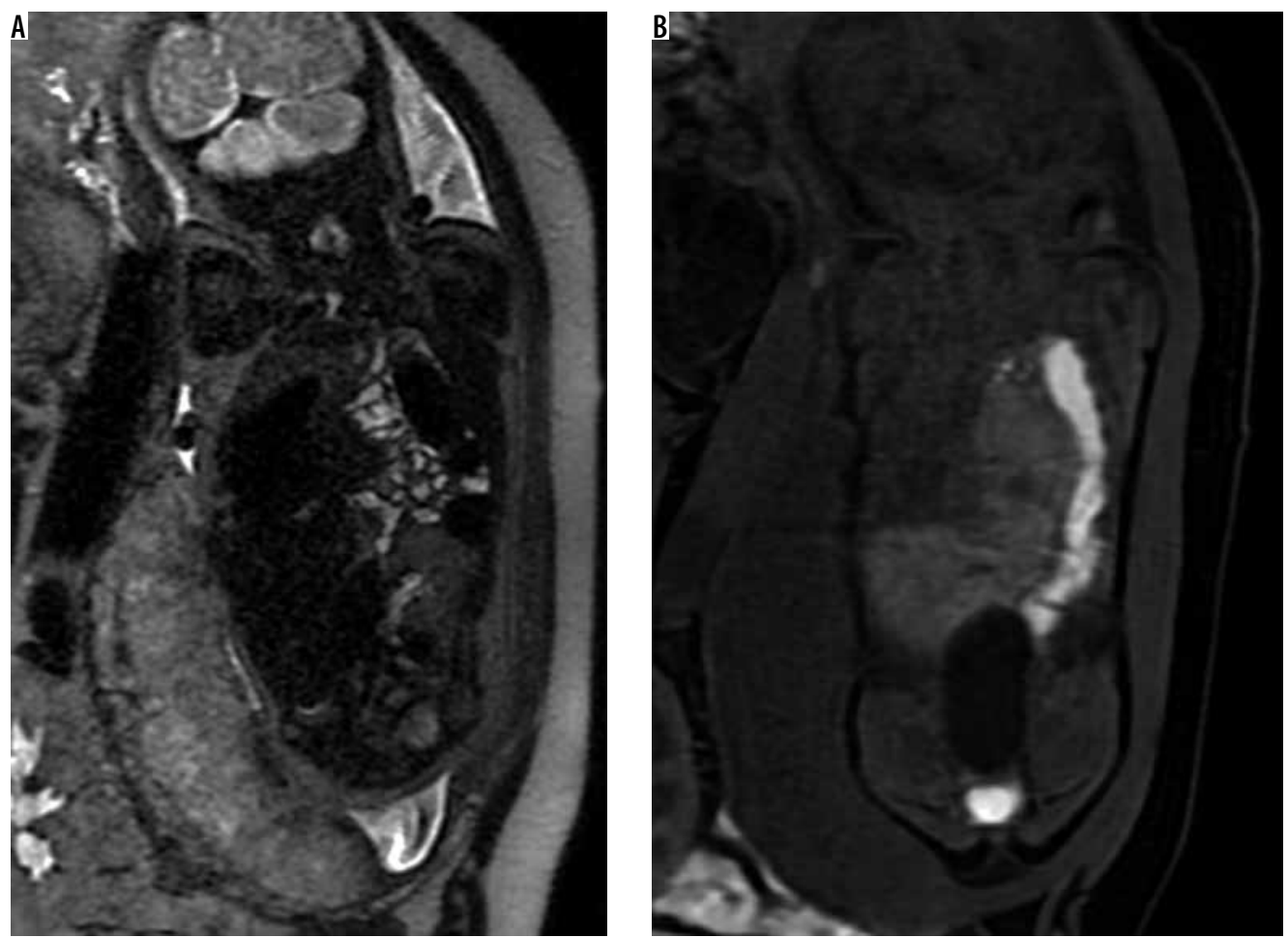

Figure 5. $39 \mathrm{Hbd}$. A) SSFSE sequence, T2-weighted images, B) LAVA. Congenital diaphragmatic hernia on the left side with small bowel (A), left liver lobe, and large bowel (B) in the chest. The heart and thymus displaced to the right hemithorax (A). The fat-saturated T1-weighted image (B) clearly depicts the liver and meconium-filled colon in the thorax

gall bladder, renal collecting systems, and bladder, are typically T2-hyperintense and T1-hypointense. Normal biliary ducts are not visible during foetal life [34]. Abnormal kidneys display increased SI on T2WI and lack

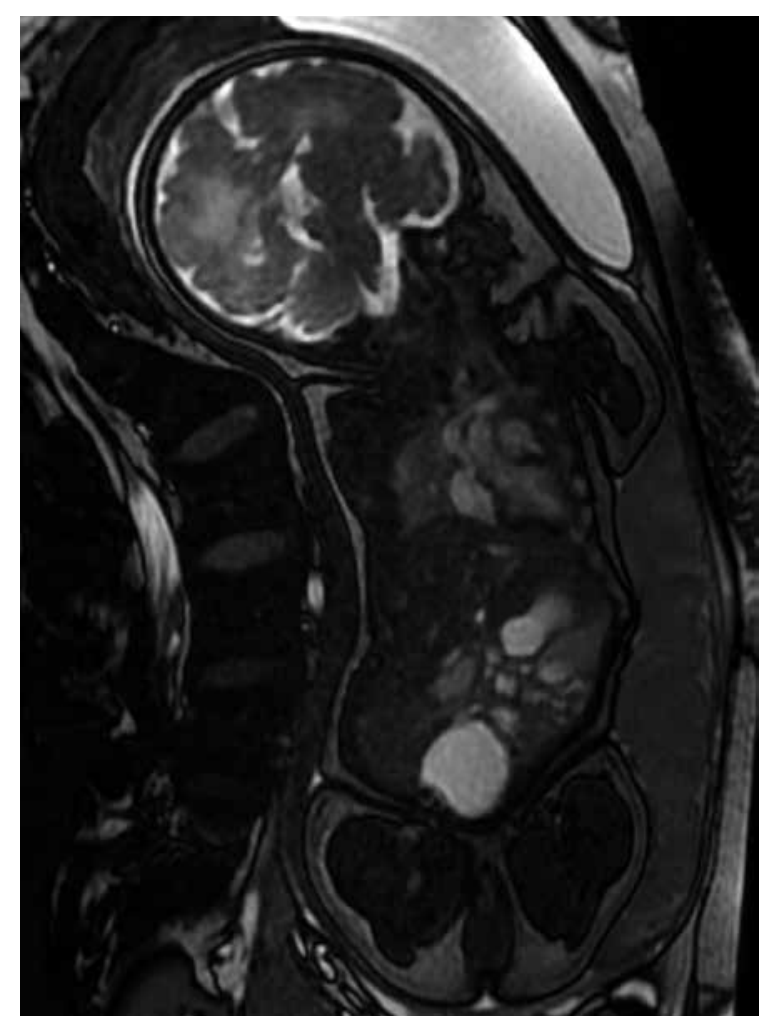

Figure 6. $36 \mathrm{Hbd}$. FIESTA/2D image with normal heart filled with "white blood" normal bright signal on DWI [26]. Conversely, in the case of an apparently missing kidney on US, MRI helps to find it in an ectopic location due to the hyperintensity of normal renal parenchyma on DWI (Figures 2B-G). Foetal MRI can assess mass lesions in the abdomen and pelvis, as in other regions of the body. Ovarian cysts, teratomas, and haemangiomas belong to the most common of them (Figure 7). The FLAIR sequence is helpful in the differentiation of physiologically fluid-filled structures and cystic lesions with thicker contents, e.g. ovarian cysts.

Finally, the development of new sequences, like GRE EPI, have allowed MRI assessment of bones that were earlier evaluated exclusively by US. This sequence reveals black calcified bone and bright cartilages (Figures 8A-B). One can observe black ossification centres appearing over time against the background of bright cartilages (Figure 8C). One can also diagnose an in utero fracture of a long bone (Figure $8 \mathrm{C}$ ).

With the increasing number of multifoetal pregnancies and increased probability of congenital anomalies in these pregnancies, we deal more and more often with twin pregnancy referrals for MRI. Also, complications specific for twin gestations (consequences of intrauterine death of one twin to another one, twin-twin transfusion syndrome (TTTS), twin anaemia-polycythaemia syndrome (TAPS), twin reversed-arterial perfusion syndrome (TRAPS), and conjoined twinning) may require MRI, e.g. for better evaluation of brain malformations in a surviving twin or to assess the possibility of separation of conjoined twins. In addition to the well-known limitations of foetal US, 
another problem in twin pregnancy is that one foetus may interfere with the attempt to evaluate another one on US, which is not a problem when using MRI [15]. The number of referrals in these cases has increased significantly, and the number of pairs of twins examined at our centre has reached 45 (Figures 9A-C).

We described above the utility of MRI in the assessment of the particular regions of the foetal body, but one of the biggest advantages of this method is its utility in detection of complex malformations and multifocal anomalies (Figure 2). It is of utmost importance in this field of diagnostic imaging to avoid the so-called "happy radiologist syndrome", i.e. the situation in which one is satisfied with the first finding and does not notice the other deviations from the norm. This does not mean that we were free from such events in the past. The example is detailed characterisation of a head and neck mass as lymphangioma and missing a sacrococcygeal teratoma, which was barely visible in one image because this region of the body was omitted, and the focus was only on the upper part. Learning from one's own mistakes is an important part of a radiologist's education.

The broad field of view of MRI and the images that appeal to the imagination of the parents and, above all, of surgeons and other specialists who will be involved in further management, before and/or after birth of a baby,

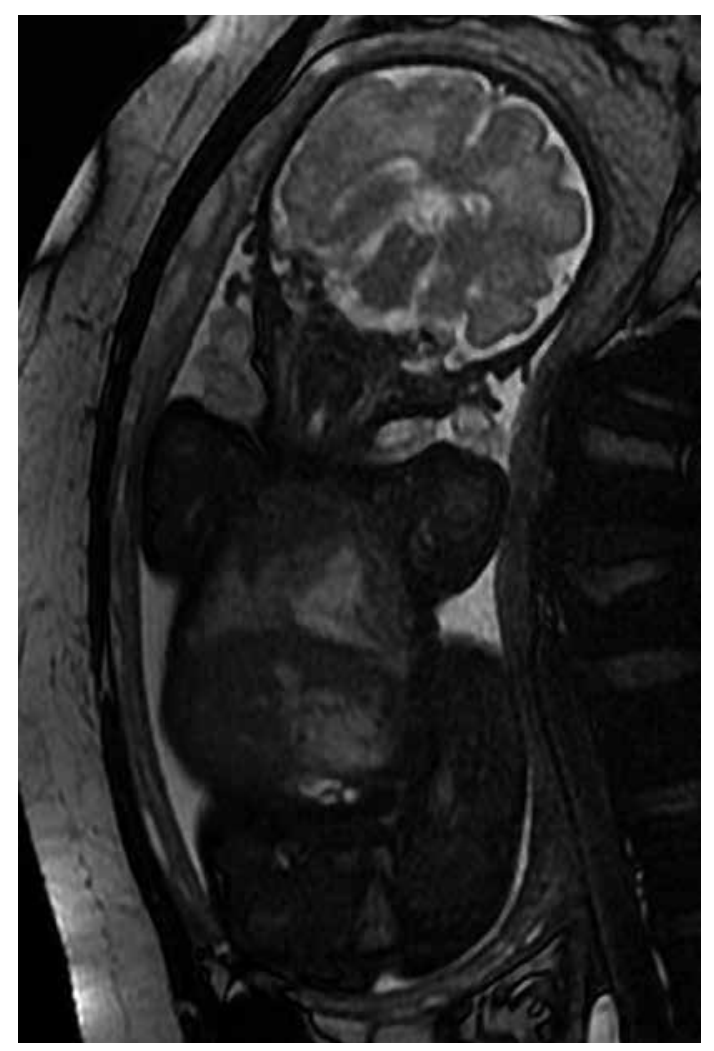

Figure 7.31 Hbd. FIESTA/2D. Haemangioma in the left lobe of the liver
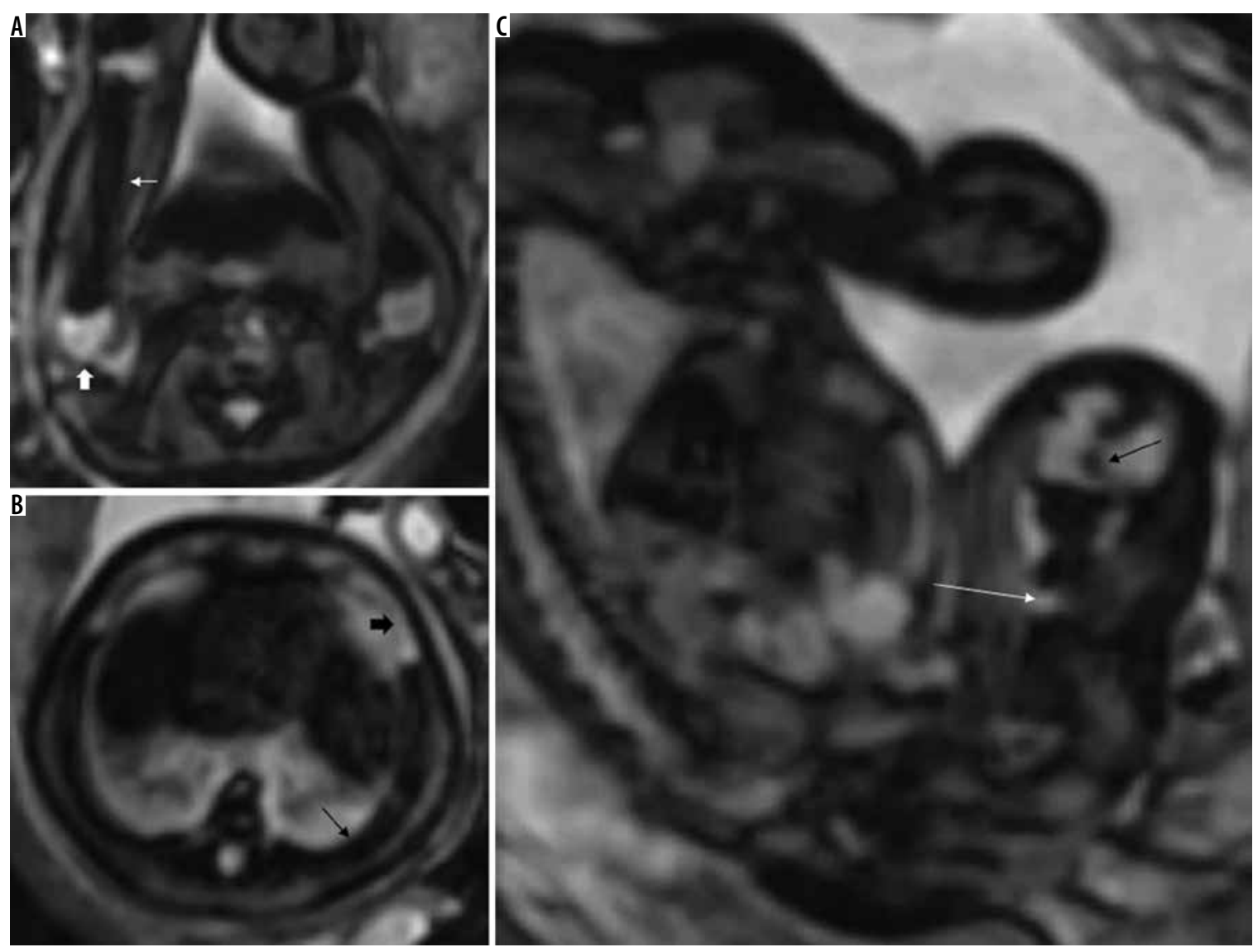

Figure 8. GRE EPI sequence nicely depicts calcified bone (black) and cartilage (bright). A, B) $36 \mathrm{Hbd}$. A) Humerus (shaft - thin arrow, cartilaginous epiphysis - thick arrow), B) rib (bony posterior part - thin arrow, cartilaginous anterior part - thick arrow). C) $35 \mathrm{Hbd}$, skeletal dysplasia: in utero fracture of the short and thick femoral shaft - thin arrow; ossification nucleus of the cartilaginous epiphysis - thick arrow) 


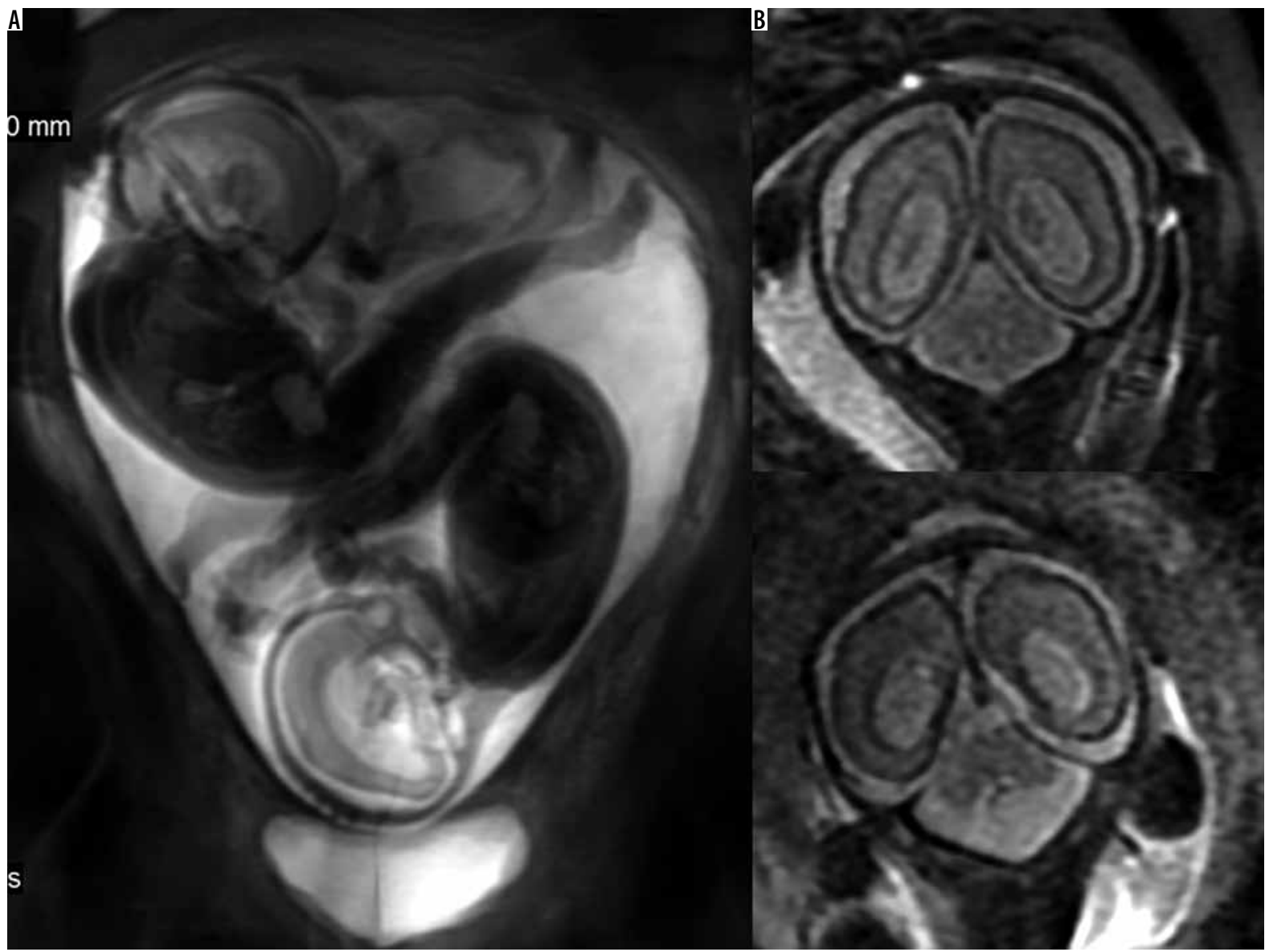

Figure 9. Dichorionic pregnancy, $24 \mathrm{Hbd}$ (A - thick slab). Ventriculomegaly was diagnosed in both twins (B, C - SSFSE sequence, T2-weighted images) on ultrasound while magnetic resonance imaging revealed also cerebellar hypoplasia in one of them (C)

are among the most important and greatest advantages of foetal MRI. And here we come to a very important point, which is the purpose of performing foetal MRI: at our centre it serves the activities of the Interdisciplinary Team for the Diagnosis and Treatment of Foetal Anomalies, which has been working for many years, bringing together specialists from various fields of perinatal medicine. The task of the interdisciplinary team is to individually assess each case of birth defects and other congenital diseases of the foetus, to establish diagnosis and treatment planning during pregnancy and after delivery, and to create and conduct a registry of birth defects and congenital tumours. The team assesses the compatibility of parental requests about pregnancy termination with the law on family planning, human embryo protection, and conditions of permissibility of abortion. The clarity of MR images increases the conviction of decision makers as to the appropriateness of their decisions. Finally, the team is an element of the care system for families affected by these problems. The team can decide to repeat MRI in the later stage of pregnancy, if necessary, as even in a facility such as ours, equipped with a specialised MR-compatible incubator for imaging neonates, the study of the foetus in utero is less stressful for the baby and is an easier logistical task than examination of the already born sick baby [10].
In conclusion, MRI at our Institute is a practically used second-line foetal imaging tool, necessary to confirm, complete, or correct sonographic diagnoses, with important impact on clinical decisions concerning management of pregnancy and of the neonate, termination of pregnancy, choice of the time, place and mode of delivery, and neonatal care, as well as on counselling. In experienced hands MRI is to date the last and the best non-invasive method of diagnosis in utero. It is frequently requested by the interdisciplinary team for foetal diagnosis and therapy and routinely performed in everyday practice.

\section{Acknowledgements}

The authors wish to thank the team of MR technologists, without whom it would be impossible to perform these examinations and without whom this manuscript would not have been created.

\section{Disclosure}

Preparation of this manuscript was supported by the internal grant of the Institute of Mother and Child in Warsaw.

The authors declare no conflict of interest. 
References

1. Wermeński K, Wagiel K, Bekiesińska-Figatowska M, et al. Magnetic resonance imaging in prenatal diagnosis. Ginekol Pol 2002; 73: 142-149.

2. Jurkiewicz E, Bekiesińska-Figatowska M, Romaniuk-Doroszewska A, et al. Brain MRI in fetuses with cardiac tumours. Neuroradiol J 2007; 20: 494-499.

3. Herman-Sucharska I, Bekiesińska-Figatowska M, Urbanik A. Fetal central nervous system malformations on MR images. Brain Dev 2009; 31: 185-199.

4. Jurkiewicz E, Bekiesińska-Figatowska M, Duczkowski M, et al. Antenatal diagnosis of the congenital craniopharyngioma. Pol J Radiol 2010; 75: 98-102.

5. Duczkowska A, Bekiesinska-Figatowska M, Herman-Sucharska I, et al. Magnetic resonance imaging in the evaluation of the fetal spinal canal contents. Brain Dev 2011; 33: 10-20.

6. Bekiesińska-Figatowska M, Herman-Sucharska I, Romaniuk-Doroszewska A, et al. Brain development of the human fetus in magnetic resonance imaging. Med Wieku Rozwoj 2010; 14: 5-14.

7. Bekiesinska-Figatowska M, Jurkiewicz E, Duczkowski M, et al. Congenital CNS tumors diagnosed on prenatal MRI. Neuroradiol J 2011; 24: 477-481.

8. de Laveaucoupet J, Bekiesińska-Figatowska M, Rutkowska M. What is the impact of fetal magnetic resonance imaging (MRI) on prenatal diagnosis of cerebral anomalies. Med Wieku Rozwoj 2011; 15 (3 Pt 2): 376-384.

9. Bekiesinska-Figatowska M, Herman-Sucharska I, Romaniuk-Doroszewska A, et al. Diagnostic problems in case of twin pregnancies: US vs. MRI study. J Perinat Med 2013; 41: 535-541.

10. Bekiesińska-Figatowska M, Herman-Sucharska I, Duczkowska A, et al. Prenatal MRI as a method of controlling fetal pathology. Ginekol Pol 2013; 84: 436-443.

11. Helwich E, Bekiesińska-Figatowska M, Bokiniec R. Recommendations regarding imaging of the central nervous system in fetuses and neonates. J Ultrason 2014; 14: 203-216.

12. Bekiesińska-Figatowska M, Brągoszewska H, Romaniuk-Doroszewska $\mathrm{A}$, et al. The role of magnetic resonance imaging in the prenatal diagnosis of cleft lip and palate. Dev Period Med 2014; 18: 27-32.

13. Bekiesinska-Figatowska M, Romaniuk-Doroszewska A, Duczkowska A, et al. Fetal MRI versus postnatal imaging in the MR-compatible incubator. Radiol Med 2016; 121: 719-728.

14. Bekiesińska-Figatowska M. Prenatalne badania metodą rezonansu magnetycznego. In: Położnictwo. Vol. 4. Diagnostyka biofizyczna i biochemiczna w medycynie perinatalnej. Bręborowicz GH, Wielgoś M (eds.). Wydawnictwo Lekarskie PZWL, Warsaw 2012; 239-247.

15. Bekiesinska-Figatowska M. MR Imaging of Multiple Gestations. In: MRI of Fetal and Maternal Diseases in Pregnancy. Masselli G (ed.). Springer International Publishing, Switzerland 2016; 231-244.

16. Bekiesińska-Figatowska M, Herman-Sucharska I, Urbanik A (eds.). Atlas MR wad płodu. Katedra Radiologii Collegium Medicum UJ i Okręgowa Izba Lekarska w Krakowie, Krakow 2013.

17. Prayer D, Malinger G, Brugger PC, et al. ISUOG Practice Guidelines: performance of fetal magnetic resonance imaging. Ultrasound Obstet Gynecol 2017; 49: 671-680.

18. Levine D. Ultrasound versus magnetic resonance imaging in fetal evaluation. Top Magn Reson Imaging 2001; 12: 25-38.
19. American College of Radiology (ACR), Society for Pediatric Radiology (SPR). ACR-SPR practice guideline for the safe and optimal performance of fetal magnetic resonance imaging (MRI). [online publication]. Reston (VA): American College of Radiology (ACR); 2010. p. 10. Available from: http://www.guidelines.gov/content.aspx?id=32509.

20. Miller E, Ben-Sira L, Constantini S, et al. Impact of prenatal magnetic resonance imaging on postnatal neurosurgical treatment. J Neurosurg 2006; 105 (3 Suppl): 203-209.

21. Brugger PC, Stuhr F, Lindner C, et al. Methods of fetal MR: beyond T2-weighted imaging. Eur J Radiol 2006; 57: 172-181.

22. Yamashita $Y$, Namimoto T, Abe $Y$, et al. MR imaging of the fetus by a HASTE sequence. Am J Roentgenol 1997; 168: 513-519.

23. Bekiesińska-Figatowska M. Fetal MRI: is it safe? J Pediatr Neuroradiol 2012; 1: 155-159.

24. Glenn OA, Barkovich AJ. Magnetic resonance imaging of the fetal brain and spine: an increasingly important tool in prenatal diagnosis, Part 1. Am J Neuroradiol 2006; 27: 1604-1611.

25. Adzick NS, Thom EA, Spong CY, et al. A randomized trial of prenatal versus postnatal repair of myelomeningocele. N Engl J Med 2011; 364: 993-1004.

26. Savelli S, Di Maurizio M, Perrone A, et al. MRI with diffusion-weighted imaging (DWI) and apparent diffusion coefficient (ADC) assessment in the evaluation of normal and abnormal fetal kidneys: preliminary experience. Prenat Diagn 2007; 27: 1104-1111.

27. Huisman TAGM. Foreword. J Pediatr Neuroradiol 2012; 1: 145.

28. Saleem SN, Said AH, Abdel-Raouf M, et al. Fetal MRI in the evaluation of fetuses referred for sonographically suspected neural tube defects (NTDs): impact on diagnosis and management decision. Neuroradiology 2009; 51: 761-772.

29. Kasprian G, Balassy C, Brugger P, et al. MRI of normal and pathological fetal lung development. Eur J Radiol 2006; 57: 261-270.

30. Hagelstein C, Weidner M, Kilian AK, et al. Repetitive MR measurements of lung volume in fetuses with congenital diaphragmatic hernia: individual development of pulmonary hypoplasia during pregnancy and calculation of weekly lung growth rates. Eur Radiol 2014; 24: 312-319.

31. Wielgoś M, Kosiński P. FETO - fetoscopic endotracheal balloon occlusion. Ginekol Pol 2014; 85: 651.

32. Donofrio MT, Moon-Grady AJ, Hornberger LK, et al. Diagnosis and treatment of fetal cardiac disease: a scientific statement from the American Heart Association. Circulation 2014; 129: 2183-2242.

33. Farhataziz N, Engels J, Ramus R, et al. Fetal MRI of urine and meconium by gestational age for the diagnosis of genitourinary and gastrointestinal abnormalities. Am J Roentgenol 2005; 184: 1891-1897.

34. Brugger PC. MRI of fetal abdomen. In: Fetal MRI. Prayer D (ed.). Springer-Verlag, Berlin 2011; 361-401. 